\title{
Periodic driving leaves deeper quantum scars
}

\author{
Controlling many-body dynamics with driven quantum scars in Rydberg \\ atom arrays \\ Authors: Dolev Bluvstein Dolev Bluvstein, Ahmed Omran, Harry Levine, Alexander \\ Keesling, Giulia Semeghini, Sepehr Ebadi, Tout T. Wang, Alexios A. Michailidis, \\ Nishad Maskara, Wen Wei Ho, Soonwon Choi, Maksym Serbyn, Markus Greiner, \\ Vladan Vuletic, Mikhail D. Lukin
}

arXiv:2012.12276

\section{Recommended with a Commentary by Ehud Altman, UC Berkeley}

When you ping a cold sample of quantum matter with neutrons or light you might expect to learn about its quantum state from the few collective modes or quasiparticles excited by the small perturbation. On the other hand if you hit the sample with a hammer, you normally expect a mess even if the hammer is designed to create a very precise deformation. The deformation produced in this way is expected to rapidly decay into a multitude of manybody excitations as the system rapidly evolves toward a new thermal state consistent with the large energy it has absorbed. For this reason it was surprising when researchers in a HarvardMIT collaboration observed collective oscillations in the dynamics of an interacting manybody atomic system initialized in certain high energy states [1]. This apparent violation of conventional notions of thermalization was soon explained using the fascinating concept of quantum scars [2]. The experiment in the recommended paper presents a new intriguing result on how the oscillations attributed to approximate scars respond to periodic parametric driving. While driving a many-body system normally leads to heating and thus more rapid decay of coherent signals, the experiment shows that a certain type of driving leads to slower entropy production and longer lived oscillations. This raises the question if driving can provide a generic mechanism to stabilize scarred subspaces or even create new protected states that do not exist in the static system.

There are clear recent reviews about general aspects of quantum scars (see for example [3]) and even an excellent commentary written for this venue [4]. Nonetheless, I want to briefly review certain aspects of the problem that are pertinent to understanding the new twist in the story that is my focus here. Quantum many body scars are understood as a protected sub-subspace spanned by a special set of rare "non-thermal" eigenstates, coexisting with the majority many-body spectrum. The scar states are "non thermal" in the sense of having sub-extensive area law entanglement entropy as in the quantum ground state and in contrast to generic many-body eigenstates with which they coexist in the spectrum. It is possible to describe the scar sub-space as a special kind of collective mode, generated by a ladder operator $Q^{\dagger}$ from a simple low entanglement "vaccuum" state: $\left|\psi_{n}\right\rangle=\left(Q^{\dagger}\right)^{n}|0\rangle$. If the operator $\left[H, Q^{\dagger}\right]-\omega_{0} Q^{\dagger}$ vanishes when acting on this subspace, then the $\left|\psi_{n}\right\rangle$ form a tower 
of equidistant energy eigenstates. This allows to view them as different occupations of a collective mode with energy $\omega_{0}$. Crucially, because the vacuum is a simple low entanglement state and the ladder operator $Q^{\dagger}$ is a sum of local terms, all the states in the tower are similarly local. So, although the scar subspace is very atypical in the many-body spectrum, it is rather natural to access and manipulate it in the lab if it exists. In particular, easily prepared product states can have a large overlap with a macroscopically occupied coherent state of the ladder operator $Q$ and thus would show persistent oscillations at the frequency $\omega_{0}$. This is an important distinction from usual low lying collective modes, which generally decay rapidly if occupied by more than a few quanta.

The existence of a truly protected scar subspace seems to be a rare special property of highly fine tuned models. Although there is a growing number of examples of such models, adding small generic perturbations to them invariably leads to a finite lifetime of the scar states. This is also the case in the Rydberg atom arrays, studied in the recommended paper and in the earlier experiment [1]. The dynamic degrees of freedom here are the atomic states on each site, which in presence of near resonant light, form effective two level systems comprising of the ground state $|\downarrow\rangle$ and excited Rydberg state $|\uparrow\rangle$. The effective hamiltonian of a single atom in the rotating frame is then just $h_{1}=-\Omega \sigma^{x}$, where $\Omega$ is the Rabi frequency. However, the repulsive interactions between Rydberg states effectively prohibit occupation of the $|\uparrow\rangle$ state in neighboring sites, leading to the so called PXP effective Hamiltonian $H=-\Omega \sum_{i} P_{i-1} \sigma_{i}^{x} P_{i+1} \equiv-\Omega \tilde{S}^{x} . \quad P_{j}$ is projection to the $|\downarrow\rangle$ on site $j$, which is needed to implement the constraint imposed by the Rydberg blockade. In spite of it's apparent simplicity, the PXP model is strongly interacting and non-integrable. It was argued that the operator $Q^{\dagger}=\frac{1}{2} \sum_{j}(-1)^{j} P_{j-1}\left(\sigma_{j}^{y}+\mathrm{i} \sigma_{j}^{z}\right) P_{j+1}$ generates an approximate scar tower leading to the observed collective oscillations at a renormalized frequency $\omega_{0} \approx 0.6 \Omega$. Within this subspace the oscillations appear as a precession affected by the effective global magnetic field coupled to $\tilde{S}^{x}$. The oscillation ultimately decays because the required scar condition $\Omega\left[\tilde{S}^{x}, Q^{\dagger}\right]\left|\psi_{n}\right\rangle=\omega_{0} Q^{\dagger}\left|\psi_{n}\right\rangle$ is closely, but not perfectly satisfied on the scar subspace. This begs the question if there is a generic way to stabilize such protected subspace and make the oscillating mode longer lived or even truly protected?

The recommended paper claims to find a route to stabilize the scars by applying a strong time periodic drive. This might appear surprising at first because a time dependent drive is generally expected to cause faster decay of coherent phenomena due to heating. Before attempting to explain what is going on, let me first give a brief summary of the main results of the experiment. A new element added to the system is a time periodic modulation of the detuning from the atomic transition, leading to a modulated $\mathrm{z}$ field in the effective model

$$
H=H_{P X P}+\left(\Delta_{0}+\Delta_{m} \cos \left(\omega_{m} t\right)\right) \sum_{j} \sigma_{j}^{z}
$$

where $\Delta_{0}, \Delta_{m}$ and $\Omega$ are all of comparable magnitude. In addition the experiment is done with wide range of lattice geometries and a decaying oscillation is observed both in one dimensional chains and in bi-partite two dimensional lattices. These are the main findings on how the oscillation responds to the periodic modulation: (1) a macroscopic oscillation between the same two classical states as in the non driven system is seen for a range of modulation frequencies $\omega_{m}$ centered around $2 \omega_{0}$. The observed oscillation frequency is however 

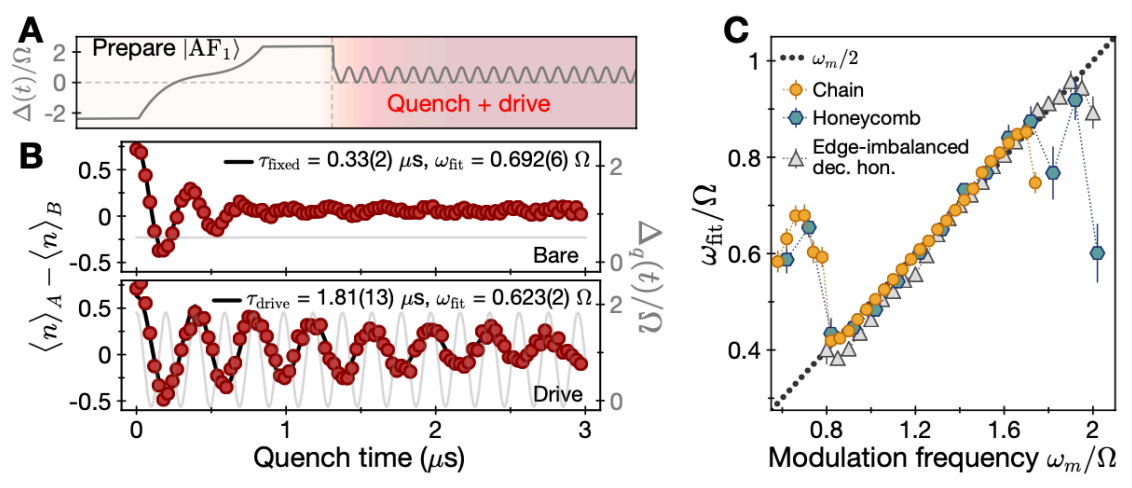

Figure 1: Summary of experimental results (taken from Fig. 3 of the recommended paper). A: The experimental protocol. B: Observed oscillations of the z-antiferromagnetic order in the non-driven and driven cases. C: Observed oscillation frequency versus modulation frequency shows locking to half of the modulation frequency.

modified and locked to exactly half of the modulation frequency. (2) The lifetime of the oscillation is increased by up to a factor of 5 in presence of the periodic driving.

One might be tempted to view these results as an example of the well known phenomenon of parametric amplification. Let's suppose for now that we can think of the observed oscillations as a resonant harmonic mode with a natural frequency $\omega_{0}$ and line-width $\gamma$. Parametric excitation of such a resonant mode using a drive with frequency $\omega_{m} \approx 2 \omega_{0}$ and strength $\lambda$ is described by a standard equation of motion for the coherent state of the mode (in a frame rotating with $\left.\omega_{m} / 2\right)$ :

$$
\dot{a}=-\mathrm{i} \delta \omega a+\mathrm{i} \lambda a^{\star}-\gamma a-u|a|^{2} a,
$$

where $\delta \omega=\omega_{0}-\omega_{m} / 2$. The nonlinearity $u$ allows the mode occupation to saturate in the amplification regime. Solving for the steady state gives the mode occupation: $|a|^{2}=$ $u^{-1}\left(\sqrt{\lambda^{2}-\delta \omega^{2}}-\gamma\right)$. Thus, for a sufficiently strong drive there is a wide regime, where a solution exists, implying a stable oscillating mode, locked to the frequency $\omega_{m} / 2$. This picture appears to give a disappointingly mundane explanation of the experimental result. Can it be correct?

One problem with this story is that it does not explain slowing down of entropy production. Since we are dealing with a closed system, the dissipation constant $\gamma$ stems from coupling to and production of excitations of the system in the space complementary to the observed collective mode. Stabilization of the coherent oscillation is not achieved by slowing this dissipation, but rather by compensating it with coherent pumping that replenishes the mode occupation. Eventually the mode can no longer be sustained when the effective bath gets too hot and the noise it produces on the collective mode exceeds the dissipation it allows. Thus it is not even clear that in absence of an external bath such a mechanism can prolong the lifetime of the amplified mode and certainly it does not slow and even accelerates entropy production in the rest of the system. It is hard to measure the entropy production rate precisely, but snapshots of the spin configurations taken as a function of time indicate a higher rate of entropy production in the non driven case. Moreover numerical simulation of the PXP model on small systems clearly show suppressed growth of the half system en- 
tanglement entropy in the driven system. These results rule out the simple amplification model. What is then the mechanism for scar stabilization?

A possible mechanism is suggested in the paper and demonstrated with a simplified model. In the proposed model the time dependent detuning term $\Delta(t)$ is applied in deltafunction pulses instead of a continuous modulation. If the strength of the pulses is tuned to exactly induce a $\pi$ rotation around the $\sigma^{z}$ axis, then the time evolution by the bare PXP hamiltonian is effectively inverted. Thus the time evolution between pulses $j$ and $j+1$ is exactly undone by the evolution from $j+1$ to $j+2$, leading to a trivial period two oscillation independent of any scar dynamics. But the presence of a scar oscillation is crucial for endowing this protocol with robustness to errors. Between two approximate $\pi$ pulses about the $\sigma^{z}$ axis induced by the pulsed drive, the scar oscillation affects an approximate $\pi$ pulse about $\sigma^{x}$. Such a sequence of alternating $\pi$ pulses about orthogonal axes is a known protocol in NMR to cancel errors in preceding pulses and stabilize a period 2 oscillation.

The proposed mechanism leaves many unanswered questions. First, it is at the same time strange and remarkable that such a control protocol, designed for a single spin may also be useful to stabilize the dynamics of a complex many-body quantum system. A specific point of concern in this regard is that the NMR sequence cancels errors in the rotation of a single spin around the Bloch sphere, while in many-body systems, some of the error stems from dissipation or entanglement with other degrees of freedom and thus has very different character. Are such errors also partially cancelled in this protocol? At a more conceptual level the protocol is engineered to utilize specific features of the dynamics of this particular system and thus might not imply a dynamical mechanism with more general application. Nonetheless the demonstrated possibility to stabilize a complex many-body state with a simple dynamic drive should certainly motivate more work to identify a possible universal mechanism that achieves this goal.

\section{References}

[1] H. Bernien et. al., Nature 551, 579-584 (2017)

[2] C. J. Turner, A. A. Michailidis, D. A. Abanin, M. Serbyn and Z. Papić, Nature Phys. 14, 745-749 (2018).

[3] M. Serbyn, D. A. Abanin, Z. Papić, arXiv:2011.09486

[4] R. Nandkishore, Journal Club for Condensed Matter Physics, April 2020. "doi = 10.36471/JCCM_April_2020_01" 